\title{
Pediatric Eosinophilic Granuloma in the Skull after Minor Head Trauma: A Case Report
} \author{
Seoul, Republic of Korea \\ Corresponding author: Hee Sup Shin \\ Department of Neurosurgery, Kyung- \\ Hee University Hospital at Gangdong, \\ Kyung Hee University School of \\ Medicine, 892 Dongnam-ro, \\ Gangdong-gu, Seoul 05278, \\ Republic of Korea \\ Tel: $+82-2-440-6090$ \\ Fax: +82-2-440-8404 \\ E-mail: realeponym@hanmail.net
}

Hwan Seok Shim, Hee Sup Shin, Seung Hwan Lee, Jun Seok Koh, Hak Cheol Ko

Department of Neurosurgery, Kyung Hee University Hospital at Gangdong, Kyung Hee University School of Medicine,

Received: July 30, 2020

Revised: Auqust 11, 2020

Accepted: August 12, 2020
Here, we report a case of eosinophilic granuloma (EG) of the skull in a pediatric patient after minor head trauma without hemorrhage or fracture. A 15 -year-old boy presented with a soft mass on the left side of his forehead. The mass was $2 \mathrm{~cm}$ in size and was associated with tenderness. It had grown rapidly in 1 month. He had been hit by a baseball on the left side of his forehead 9 months before. Cerebral computed tomography revealed a 2-cm-sized mass in the frontal bone region on the left side with a lytic bony defect. This mass was wellenhanced on cerebral magnetic resonance imaging, and the margin was well-demarcated without intracerebral infiltration. The mass was removed surgically. Total excision was performed through craniectomy around the mass, and the bony defect was reconstructed using bone cement. Three years postoperatively, there was no local or distant recurrence. Minor trauma without hemorrhage or fracture can trigger the development of EG. In pediatric patients, clinicians should be aware of a possible association between solitary EG of the skull and minor head trauma.

Key Words: Eosinophilic granuloma; Head injuries, closed; Pediatric; Surgical procedures, operative

\section{INTRODUCTION}

Eosinophilic granuloma (EG), the mildest form of Langerhans cell histiocytosis (LCH), is a benign and unifocal bone lesion. Pathologic characteristics of EG are lysis of normal bony tissue with proliferation of bone-marrow-derived antigen-presenting cells $^{3)}$. EG mostly occurs in children, adolescents, and young adults, and the most commonly involved site is the skull ${ }^{8)}$. The presenting symptom and prognosis of EG are usually benign; however, the spectrum of clinical course varies from spontaneous regression to relapse, and the etiology is also heterogeneous ${ }^{3)}$. Herein, we report a case of EG after minor head trauma in a pediatric patient treated with surgical excision successfully.

\section{CASE REPORT}

A 15-year-old boy had visited the emergency room after head trauma. He had been hit by a baseball on the left side of his forehead. Cerebral computed tomography (CT) had shown no definite intracranial hemorrhage or skull fracture (Fig. 1), and he had recovered without abnormal findings or neurologic symptoms. Nine months after the head trauma, he visited our clinic because of a soft mass with tenderness on the left side of his forehead. He had noticed the mass 1 month before, and the mass had grown rapidly to $2 \mathrm{~cm}$ in size. The location of the mass was consistent with the site of the head trauma. Cerebral CT revealed a 2-cm-sized mass in the frontal bone region on the left side with a lytic bony defect (Fig. 2). This mass was well-enhanced on cerebral magnetic resonance imaging, and the margin was well-demarcated without intracerebral infiltration (Fig. 3). The results of the laboratory blood test, including the erythrocyte sedimentation rate and C-reactive
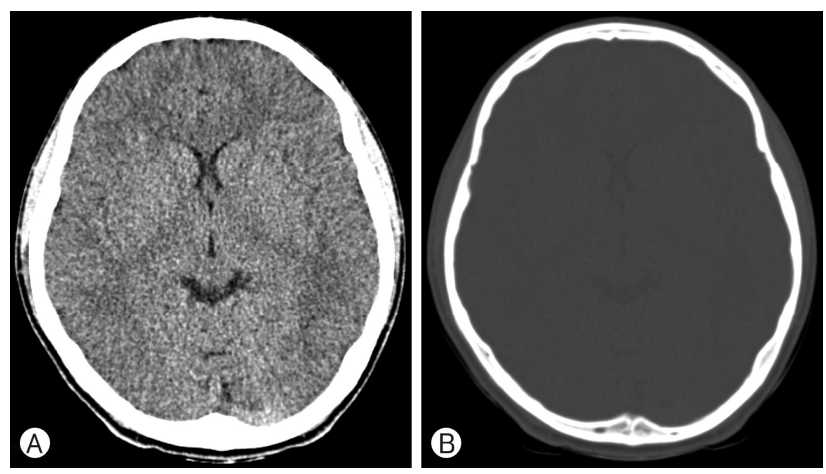

Fig. 1. Cerebral computed tomography $(\mathrm{CT})$ after minor head trauma. The CT scans show no definite intracranial hemorrhage (A) or skull fracture $(B)$. 

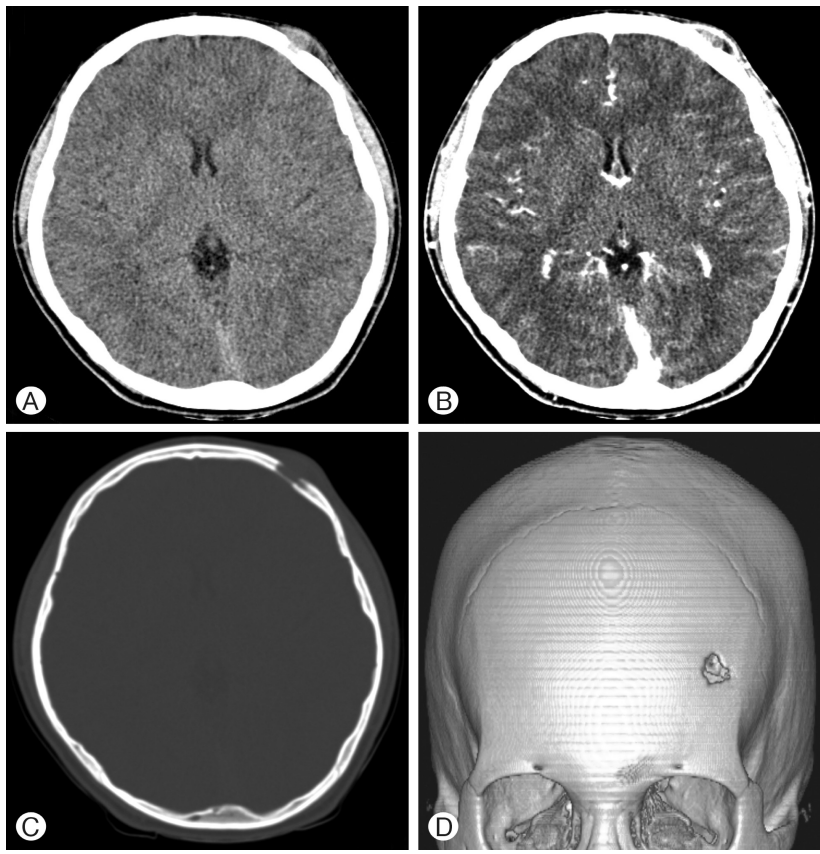

Fig. 2. Cerebral computed tomography (CT) before surgery. CT scans show a 2-cm-sized mass in the frontal bone region on the left side (A), and this mass is well-enhanced (B). The lytic bony defect is seen under the protruded tumor in the bone window setting $(\mathrm{C})$ and 3-dimensional reconstruction image (D).
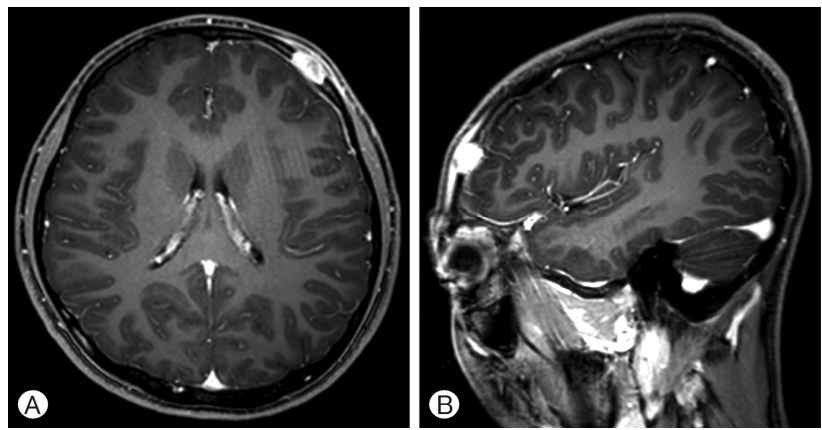

Fig. 3. Enhanced magnetic resonance image (MRI) before surgery. MRI shows an enhanced bone mass with a well-demarcated margin (A, B). There is no definite evidence of dural invasion. protein, were normal, except for mild eosinophilia. Because the patient complained of tenderness and rapid growth of the mass, we decided to perform surgery for excision of the mass.

The patient was operated under general anesthesia. The galeal layer was dissected carefully following skin incision at the hairline. After reflection of the scalp flap, a reddish mass was exposed (Fig. 4A). The mass protruded to the extracranial space from the base of the lytic bony space. We excised the protruded mass first (Fig. 4B) and then removed the base of the mass with a bony flap around the mass (Fig. 4C). We confirmed that the dura mater was intact without any tumor invasion. The tumor was removed successfully, and the bony defect was reconstructed using bone cement. Postoperative cerebral CT showed complete removal of the mass (Fig. 5), and the patient recovered without procedure-related complications.

Histopathology showed clusters of oval Langerhans' cells and a large number of eosinophils and osteoclast-like giant cells (Fig. 6A, B). In immunohistochemical staining, the majority of cells showed immunoreactivity for CD1a (Fig. 6C). These findings were consistent with $\mathrm{LCH}$. We referred the patient to a pediatrician for the work-up of $\mathrm{LCH}$. He underwent chest and abdomen CT, positron emission tomography/CT, and bone scintigraphy. The results of imaging studies were unremarkable. During a follow-up period of 3 years, the patient had no neurologic abnormalities. Cerebral CT performed 2 years postoperatively showed no recurrence.

\section{DISCUSSION}

$\mathrm{LCH}$ is a rare disease entity of the unifocal or multifocal disorder of the bone or soft tissue. Its incidence in childhood is regarded as 5.4 to 8.9 cases per million children per year ${ }^{10)}$. This disease may occur in any age group, but children and young adults are most often affected. The clinical presentation varies in organ involvement as skin only, monostotic, polyostotic, multi-system, and organ dysfunction ${ }^{3)}$. Among all types of LCH, EG is the mildest form ${ }^{8}$, which constitutes $70 \%$ of all LCH cases $^{4}$. EG can involve multiple bony lesions; however, single lesions are more common. The most frequently affected site is the skull of the lytic lesion, followed by the mandible,
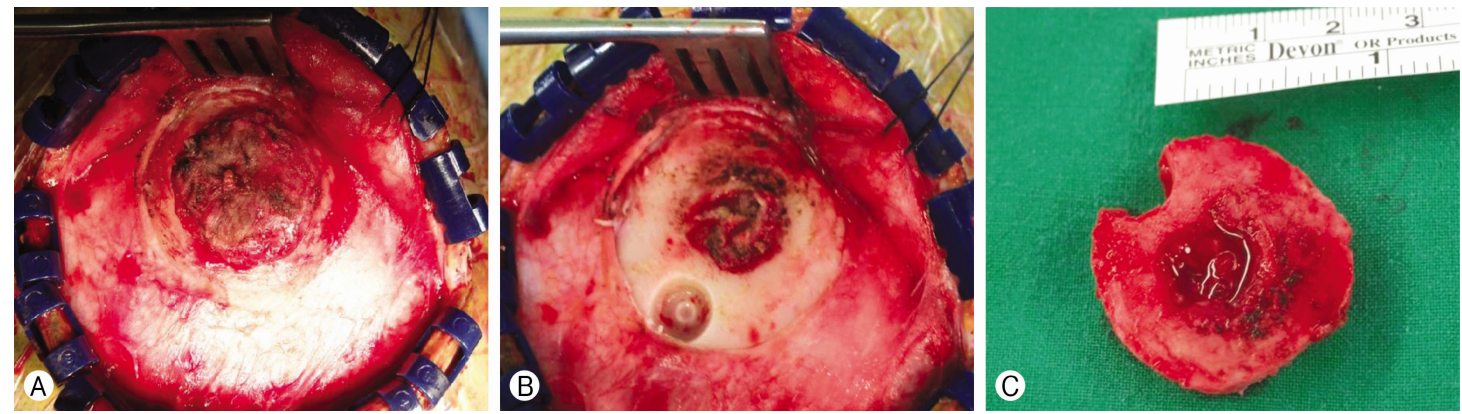

Fig. 4. Surgical removal of the mass. When the skin flap is reflected, a reddish mass is exposed (A). The mass is protruding to the extracranial space from the base of the lytic bony space. We excised the protruded mass first (B) and then removed the base of the mass with a bone flap around the mass (C). 

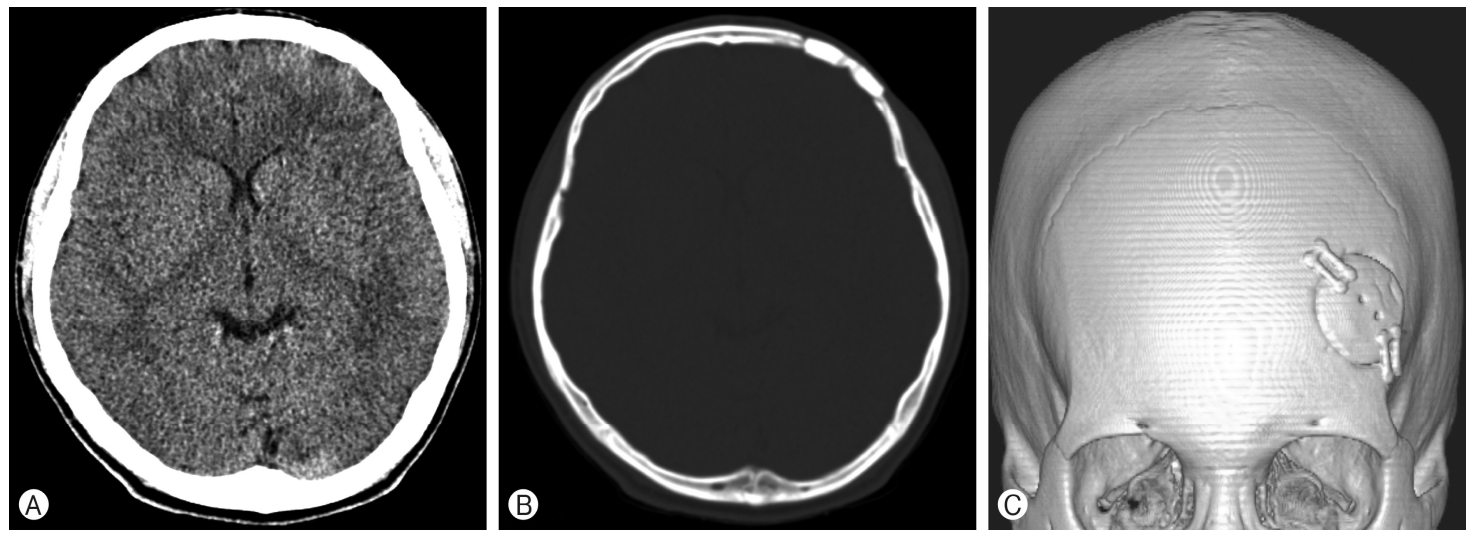

Fig. 5. Cerebral computed tomography after surgery. The mass is removed completely without procedure-related complications (A). The bony defect is reconstructed with bone cement $(B, C)$.
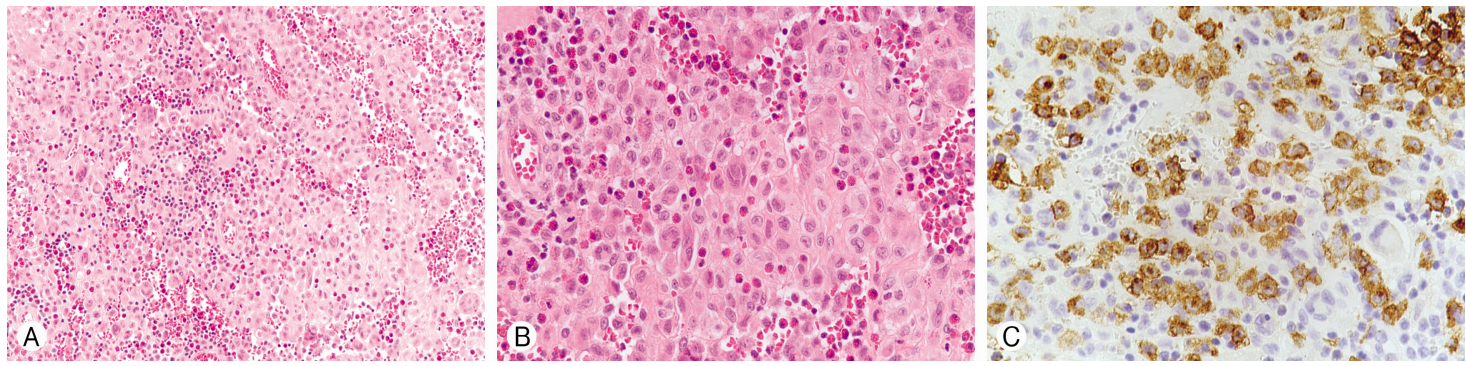

Fig. 6. Histopathological and immunohistochemical stains. In hematoxylin and eosin stain (H\&E), the image shows clusters of Langerhans cells and a large number of eosinophils and osteoclast-like giant cells (A, H\&E, $\times 200$ magnification). The high-power view shows distinctive morphology of intermediate- sized cells with grooved, folded, indented, or lobed nuclei, as well as fine chromatin, inconspicuous nucleoli, and thin nuclear membranes (B, H\&E, $\times 400$ magnification). In immunohistochemical stain, majority of cells show immunoreactivity for CDla (C).

spine, ribs, and long bones ${ }^{2,18)}$. In cases of skull lesions, the most common symptom is a gradually growing mass with tenderness, particularly in the frontal and parietal bone regions. The typical radiologic findings are punched-out, sharply defined, lytic lesions of the skull. Histologically, infiltration of Langerhans' cells, presence of eosinophilic and neutrophilic granulocytes, and granuloma formation are diagnostic. The clinical course is usually benign, and spontaneous regression may occur ${ }^{5,17)}$.

Although the exact etiology of EG is not fully understood, an abnormal inflammatory reaction, autoimmune disease, and excessive proliferation of Langerhans' cell are regarded as possible etiologic factors. Head trauma can also be considered as an etiologic factor. Some large case series documented that $33 \%$ to $50 \%$ of solitary skull EG patients had a history of head trauma ${ }^{1,6,19)}$, and minor head trauma without definite hemorrhage or skull fracture could also result in solitary $\mathrm{EG}^{9)}$. The interval from the trauma to the diagnosis is relatively short and ranges from 1 week to a few weeks, ${ }^{9,11,12,14)}$. In the present case, the patient had minor head trauma without hemorrhage or fracture before EG development, and the time interval of 9 months between the trauma and the diagnosis was quite long compared to previous reports. It is uncertain whether head trauma plays a key role in EG occurrence; however, it can be a possible mechanism for EG occurrence that triggers the amplification of abnormal inflammatory reactions at the local trauma site ${ }^{9}$.

Surgical excision is recommended for the treatment of solitary EG of the skull. The total excision through craniectomy around the mass is the most common form of surgical excision. In some cases, curettage of the lesion can be considered. Radiotherapy or chemotherapy has been recommended for multiple and recurrent lesions in some reports ${ }^{13,15,16,19)}$. Spontaneous regression has also been reported ${ }^{5,17)}$. Furthermore, lesions decrease in size 1 to 3 months after the diagnosis and disappear in a period of 3 to 19 months. In cases of decreasing size during the follow-up period, spontaneous regression can be expected. Longer follow-ups have revealed a local recurrence rate of $6 \%$ and a distant recurrence rate of $22 \% \%^{7,19,20)}$. Therefore, followup evaluations for several years have been recommended.

\section{CONCLUSION}

The exact etiology of EG is unclear. Head trauma is regarded as an etiology. In the present case, minor trauma without hemorrhage or fracture can trigger the development of EG. The interval 
from the trauma to EG occurrence was 9 months, and this was quite a long period compared to previous reports. For pediatric patients, clinicians should be aware of a possible association between solitary EG of the skull and minor head trauma.

\section{CONFLICTS OF INTEREST}

No potential conflict of interest relevant to this article was reported.

\section{REFERENCES}

1. Arseni C, Dănăilă L, Constantinescu A: Cranial eosinophilic granuloma. Neurochirurgia (Stuttg) 20:189-199, 1977

2. Azouz EM, Saigal G, Rodriguez MM, Podda A: Langerhans' cell histiocytosis: Pathology, imaging and treatment of skeletal involvement. Pediatr Radiol 35:103-115, 2005

3. Bernstrand C, Sandstedt B, Ahström L, Henter JI: Long-term follow-up of Langerhans cell histiocytosis: 39 years' experience at a single centre. Acta Paediatr 94:1073-1084, 2005

4. Chung EM, Murphey MD, Specht CS, Cube R, Smirniotopoulos JG: From the Archives of the AFIP. Pediatric orbit tumors and tumorlike lesions: Osseous lesions of the orbit. Radiographics 28: 1193-1214, 2008

5. De Angulo G, Nair S, Lee V, Khatib Z, Ragheb J, Sandberg DI: Nonoperative management of solitary eosinophilic granulomas of the calvaria. J Neurosurg Pediatr 12:1-5, 2013

6. Fox JD, Knighton RS: Diagnosis and treatment of eosinophilic granuloma of skull. J Am Med Assoc 162:1294-1297, 1956

7. Hasturk AE, Basmaci M: Multifocal extradural and intradural eosinophilic granuloma. J Craniofac Surg 24:e214-e216, 2013

8. Kaul R, Gupta N, Gupta S, Gupta M: Eosinophilic granuloma of skull bone. J Cytol 26:156-157, 2009
9. Kim YJ, Jo KW: Rapid growing eosinophilic granuloma in skull after minor trauma. Korean J Neurotrauma 11:22-25, 2015

10. Laurencikas E, Gavhed D, Stålemark H, van't Hooft I, Prayer $\mathrm{D}$, Grois $\mathrm{N}$, et al.: Incidence and pattern of radiological central nervous system Langerhans cell histiocytosis in children: A population based study. Pediatr Blood Cancer 56:250-257, 2011

11. Lee KW, McLeary MS, Zuppan CW, Won DJ: Langerhans' cell histiocytosis presenting with an intracranial epidural hematoma. Pediatr Radiol 30:326-328, 2000

12. Lee YS, Kwon JT, Park YS: Eosinophilic granuloma presenting as an epidural hematoma and cyst. J Korean Neurosurg Soc 43: 304-306, 2008

13. Leonidas JC, Guelfguat M, Valderrama E: Langerhans' cell histiocytosis. Lancet 361:1293-1295, 2003

14. Manaka S, Izawa M, Nawata H: Skull tumor simulating sinus pericranii. Case report. J Neurosurg 46:671-673, 1977

15. Martínez-Lage JF, Bermúdez M, Martínez-Barba E, Fuster JL, Poza M: Epidural hematoma from a cranial eosinophilic granuloma. Childs Nerv Syst 18:74-76, 2002

16. Martinez-Lage JF, Poza M, Cartagena J, Vicente JP, Biec F, de las Heras M: Solitary eosinophilic granuloma of the pediatric skull and spine. The role of surgery. Childs Nerv Syst 7:448451, 1991

17. Oliveira M, Steinbok P, Wu J, Heran N, Cochrane D: Spontaneous resolution of calvarial eosinophilic granuloma in children. Pediatr Neurosurg 38:247-252, 2003

18. Park SH, Park J, Hwang JH, Hwang SK, Hamm IS, Park YM: Eosinophilic granuloma of the skull: A retrospective analysis. Pediatr Neurosurg 43:97-101, 2007

19. Rawlings CE, $3^{\text {rd }}$, Wilkins RH: Solitary eosinophilic granuloma of the skull. Neurosurgery 15:155-161, 1984

20. Sauerborn D, Pajić-Penavić I, Stojadinović T: Eosinophilic granuloma of the temporal bone in an adult: controversies in the management. Coll Antropol 36 Suppl 2:163-166, 2012 